\section{Desempenho do exame citológico e da captura híbrida II no rastreamento de lesões intraepiteliais escamosas de alto grau em mulheres HIV+}

\author{
Performance by cytology and hybrid capture II in \\ screening for high-grade squamous intraepithelial \\ lesions in women with HIV
}

Letícia Martins Raposo 1,2

Luciane Velasque 2,3

Paula Mendes Luz 3

Ruth Khalili Friedman 3

Andrea Cytryn 3

Angela Cristina Vasconcelos de Andrade 3

Tazio Vanni 4

Pedro E. A. A. Brasil 3

Fabio Russomano 5

Valdiléa Gonçalves Veloso 3

Beatriz Grinsztejn 3

Claudio José Struchiner ${ }^{1}$

\begin{abstract}
${ }_{1}^{1}$ Programa de Computação Científica, Fundação Oswaldo Cruz, Rio de Janeiro, Brasil.

2 Departamento de Matemática e Estatística, Universidade Federal do Estado do Rio de Janeiro, Rio de Janeiro, Brasil. 3 Instituto de Pesquisa Clínica Evandro Chagas, Fundação Oswaldo Cruz, Rio de Janeiro, Brasil. ${ }^{4}$ London School of Hygiene and Tropical Medicine, London, U.K.

5 Instituto Fernandes Figueira, Fundação Oswaldo Cruz, Rio de Janeiro, Brasil.

Correspondência P. M. Luz Instituto de Pesquisa Clínica Evandro Chagas, Fundação Oswaldo Cruz. Av. Brasil 4365, Rio de

Abstract

$H I V$-infected women are at increased risk of developing high-grade squamous intraepithelial lesions (HSIL), the precursor lesions for cervical cancer. This study estimated and compared the performance of cytology and hybrid capture II in screening for precursor lesions of cervical cancer among HIV-infected women. The study population consisted of women from the open prospective cohort at the Evandro Chagas Clinical Research Institute, Oswaldo Cruz Foundation (IPEC/Fiocruz). Colposcopy and histology were considered jointly in defining the gold standard. Cytology showed $31.8 \%$ sensitivity and 95.5\% specificity, while hybrid capture II showed higher sensitivity (100\%) and lower specificity (52\%). The positive likelihood ratio was 7.1 for cytology and 2.1 for hybrid capture II, while the negative likelihood ratio was 0.7 for cytology and 0.0 for hybrid capture II.
\end{abstract} Janeiro, RJ 21045-900, Brasil. paula.luz@ipec.fiocruz.br
Uterine Cervical Neoplasms; HIV; Women

\section{Introdução}

O câncer do colo do útero é a segunda neoplasia mais frequente entre mulheres 1 . O papiloma vírus humano (HPV) do tipo oncogênico é uma causa necessária para o desenvolvimento do câncer do colo do útero 2,3,4. As lesões intraepiteliais escamosas de alto grau (high grade squamous intraepithelial lesions - HSIL) são atipias do epitélio cervical precursoras do câncer do colo do útero que podem ser rastreadas de diversas formas 5 . Na maioria dos países desenvolvidos e em desenvolvimento, a prevenção é feita pelo rastreamento das HSIL durante exame citológico, ou exame de Papanicolaou, seguido da colposcopia e tratamento das lesões confirmadas. Tal estratégia determinou, nas últimas décadas, uma redução da incidência e mortalidade do câncer do colo do útero, especialmente em países desenvolvidos onde a taxa de cobertura desse exame de rastreio é alta ${ }^{6}$. Atualmente, estão disponíveis outros métodos para o rastreamento das HSIL como, por exemplo, exame citológico em meio líquido, exames para detecção de DNA de HPV oncogênico, inspeção visual com ácido acético, dentre outras. É possível que diferentes técnicas de rastreamento sejam mais adequadas em algum contexto específico em razão de capacidade diagnóstica e custos diferenciados.

As recomendações com relação ao rastreamento das HSIL em mulheres infectadas pelo vírus da imunodeficiência humana (HIV+) estão 
atualmente sob avaliação 7,8. Diversos estudos demonstram que mulheres HIV+, e em particular as mulheres com imunossupressão, apresentam risco aumentado de infecção pelo HPV 3,9,10. Mulheres HIV+ também apresentam um risco aumentado de infecção por HPV oncogênico, de infecção por múltiplos HPV e de infecção persistente, sendo o risco ainda maior para as mulheres com acentuada imunossupressão 3,9,10. Além disso, a mulher HIV+ tem maior risco de desenvolver HSIL e câncer do colo do útero 7,10,11,12. A utilização do tratamento antirretroviral altamente potente (highly active antiretroviral therapy - HAART) determinou dramática redução da morbidade e mortalidade por HIV/AIDS em países desenvolvidos 13 e em desenvolvimento, em particular o Brasil 14,15. Sobrevidas prolongadas possibilitam o desenvolvimento de doenças com período de latência prolongado, como o câncer do colo do útero 16. Ainda não há um consenso sobre o efeito do HAART na evolução da infecção pelo HPV. O HAART induz a reconstituição imune possivelmente reduzindo a persistência da infecção pelo HPV e/ ou a progressão das HSIL 17,18. Por outro lado, o aumento da expectativa de vida das mulheres HIV+ resultante do uso do HAART pode, indiretamente, significar um aumento da incidência do câncer do colo do útero, particularmente em locais onde a taxa de cobertura do rastreamento é inadequada 19.

Diversos estudos estimam o desempenho de métodos de rastreamento das lesões precursoras do câncer do colo do útero na população geral 20,21,22,23. Não há, até o presente momento, estudos avaliando o desempenho de métodos de rastreamento das lesões precursoras em mulheres HIV+ em nosso meio. Neste trabalho, estimamos e comparamos o desempenho do exame citológico e da captura híbrida II no rastreamento das lesões precursoras do câncer do colo do útero em mulheres HIV+ do Rio de Janeiro, Brasil. Ainda, avaliamos o impacto do estado imunológico da mulher no desempenho dos exames de rastreamento.

\section{Métodos}

\section{A coorte: população e procedimentos}

Com o intuito de estudar a história natural da infecção pelo HIV em mulheres, uma coorte prospectiva aberta foi estabelecida, em 1996, no Instituto de Pesquisa Clínica Evandro Chagas, Fundação Oswaldo Cruz (IPEC/Fiocruz). Critérios de elegibilidade para entrada na coorte incluem confirmação do status sorológico de infecção pelo HIV e Termo de Consentimento Livre e Esclarecido assinado que confirme o desejo em participar da coorte. As participantes são incluídas independentemente do grau de imunossupressão ou do uso de medicações. As visitas ocorrem periodicamente, a cada seis meses, quando um questionário estruturado é administrado e a consulta ginecológica é realizada. O questionário abrange questões sociodemográficas, de história sexual e reprodutiva, além da história clínica no que diz respeito ao HIV e ao câncer do colo do útero e seus precursores. A consulta ginecológica compreende um exame ginecológico completo com coleta de espécime cervical para exame citológico. As mulheres são acompanhadas no Ambulatório Integrado de Ginecologia e Infectologia do IPEC/Fiocruz e no Setor de Patologia Cervical do Instituto de Fernandes Figueira (IFF/Fiocruz). O estudo foi aprovado pelo Comitê de Ética em Pesquisa do IPEC/Fiocruz.

No momento da inclusão na coorte, as mulheres tiveram espécime cervical colhido para realização dos exames citológico (esfregaço em lâmina fixada pelo álcool), de detecção de DNA de HPV pela técnica de captura híbrida II (Qiagen, Gaithersburg, Estados Unidos), e colposcópico. A obtenção de espécimes histológicos foi realizada quando indicada conforme recomendações brasileiras 24 . Esses exames foram realizados de maneira não cega, isto é, os examinadores tinham conhecimento do resultado do exame citológico ao realizarem a colposcopia, sendo ele verdadeiro para o exame histopatológico. Maiores detalhes sobre outros procedimentos e análises epidemiológicas relacionadas à coorte podem ser encontradas na literatura 25,26 .

Entre maio de 1996 e dezembro de 2007, foram incluídas 731 mulheres HIV+ na coorte. Para tal análise, foram considerados os seguintes critérios de inclusão: (i) já ter iniciado atividade sexual; (ii) não ter realizado tratamento prévio para HSIL; e (iii) não ter realizado histerectomia total. Setecentas mulheres HIV+ preencheram os critérios de inclusão. Após aplicar os critérios de exclusão, 222 mulheres foram incluídas na análise (Figura 1).

Os resultados citológicos e colposcópicos foram classificados de acordo com o Sistema de Bethesda de 198927 e com as regras da Federação Internacional de Patologia Cervical e Colposcopia revisadas em Barcelona, em 2002 28, respectivamente. A classificação histológica utilizada é análoga ao Sistema de Bethesda em que NIC 2 ou 3 (NIC2+) corresponde a HSIL. A captura híbrida II é o método laboratorial mais amplamente utilizado para a detecção da presença de DNA de determinados tipos de HPV. Esse exame fornece resultado em função da emissão de luz que é medida em unidades de luz relativa (RLU) que é proporcional à quantidade de DNA presente. A 


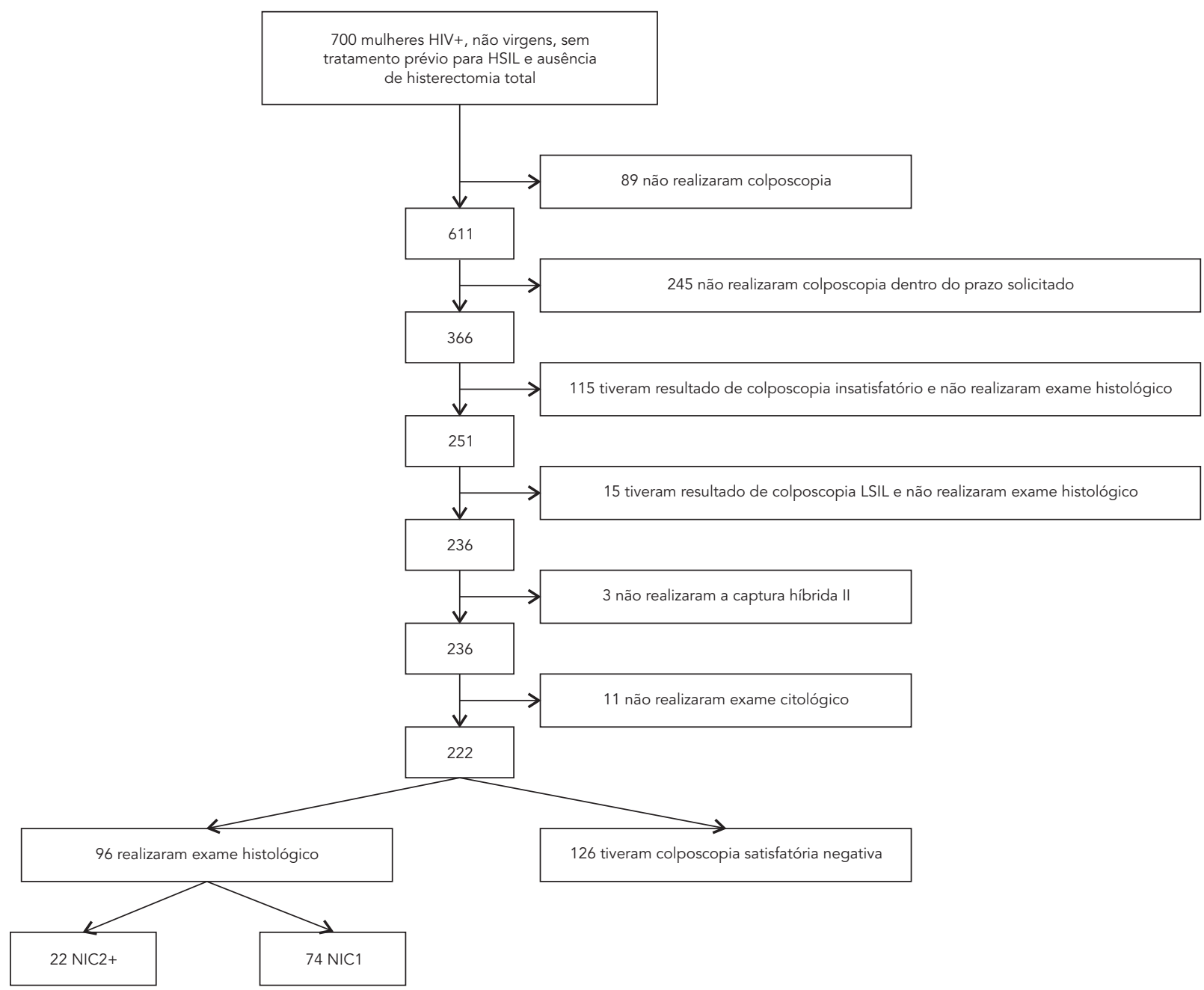

HSIL: lesão intraepitelial escamosa de alto grau; LSIL: lesão intraepitelial escamosa de baixo grau.

intensidade da luz emitida denota a presença ou ausência de DNA de HPV nos espécimes. Utilizamos o ponto de corte de RLU > 1, sugerido pelo fabricante, para o resultado positivo, indicando a presença de qualquer tipo de HPV. Foram utilizadas sondas para detecção de HPV não oncogênico (HPV 6, 11, 42, 43 e 44) e oncogênico (HPV 16, $18,31,33,35,39,45,51,52,56,58,59$ e 68$)$.

A avaliação imunológica das mulheres foi feita pela contagem de linfócitos T CD4+ (células/ $\mathrm{mm}^{3}$ ), obtida em prontuário. O estado imunológico no momento da inclusão na coorte foi definido como aquele observado em intervalo de até três meses da data da entrada na coorte. $\mathrm{O}$ CD4+ Nadir representa o grau de imunossupressão máxima apresentada pela paciente desde o diagnóstico de HIV até três meses após a data da entrada na coorte.

\section{Definição do diagnóstico segundo teste de referência}

O diagnóstico definitivo da presença de HSIL ou câncer foi dado pelo laudo histológico indicando NIC2+. Mulheres com diagnóstico histológico indicando NIC1 ou negativo ou com colposcopia 
satisfatória e negativa foram consideradas negativas para HSIL ou câncer.

\section{Plano de análise}

O desempenho do exame citológico e da captura híbrida II foi avaliado assumindo a combinação dos resultados histológicos e colposcópicos na definição do padrão ouro, conforme descrito acima. A análise do desempenho compreendeu o cálculo da sensibilidade, especificidade, do valor preditivo positivo e negativo, e das razões de verossimilhança para o teste positivo e negativo, com seus respectivos intervalos de $95 \%$ de confiança (IC95\%) 29. A chance (ou odds) foi calculada pela razão entre duas probabilidades complementares. A chance pós-teste foi calculada pelo produto da chance pós-teste pela razão de verossimilhança 30 . Dois gráficos foram utilizados para a visualização da comparação dos testes 30 . Todas as análises foram realizadas com a versão 2.10 do programa R (The R Foundation for Statistical Computing, Viena, Áustria; http:/ / www.r-project.org) 31,32.

\section{Resultados}

A idade das mulheres variou de 16 a 60 anos, com média de 32,1 anos (desvio-padrão-DP 7,8 anos) e mediana de 32 anos (intervalo interquartílico: 26-38 anos). Conforme mostra a Tabela 1, a maioria das mulheres era não branca $(54,9 \%)$, solteira $(54,5 \%)$, e apresentava entre uma e três gestações anteriores $(69,4 \%)$. Com relação ao estado imunológico das pacientes, no momento da inclusão na coorte, $75,5 \%$ das mulheres apresentaram CD4+ $\geq 200$ células $/ \mathrm{mm}^{3}$ [média 395,0 células/ $\mathrm{mm}^{3}$ (DP 289,4 células/mm³), mediana 347,5 células $/ \mathrm{mm}^{3}$ (intervalo interquartílico: 202,2-

Tabela 1

Características demográficas e clínicas das 222 mulheres HIV+ incluídas na análise.

\begin{tabular}{|c|c|c|c|c|c|c|}
\hline \multirow[t]{3}{*}{ Características } & \multicolumn{4}{|c|}{ Padrão ouro } & \multicolumn{2}{|c|}{ Total } \\
\hline & \multicolumn{2}{|c|}{ Neg/NIC1 } & \multicolumn{2}{|c|}{ NIC2+ } & \multirow[b]{2}{*}{$\mathrm{n}$} & \multirow[b]{2}{*}{$\%$} \\
\hline & $\mathbf{n}$ & $\%$ & n & $\%$ & & \\
\hline \multicolumn{7}{|l|}{ Raça } \\
\hline Branca & 90 & 45,0 & 10 & 45,4 & 100 & 45,0 \\
\hline Não branca & 110 & 55,0 & 12 & 54,6 & 122 & 55,0 \\
\hline \multicolumn{7}{|l|}{ Escolaridade } \\
\hline Sem escolaridade & 4 & 2,0 & 0 & 0,0 & 4 & 1,8 \\
\hline Primeiro grau (incompleto ou completo) & 115 & 57,5 & 12 & 54,5 & 127 & 57,2 \\
\hline Segundo grau (incompleto ou completo) & 63 & 31,5 & 8 & 36,4 & 71 & 32,0 \\
\hline Terceiro grau (incompleto ou completo) & 18 & 9,0 & 2 & 9,1 & 20 & 9,0 \\
\hline \multicolumn{7}{|l|}{ Casada/Vive com parceiro } \\
\hline $\operatorname{Sim}$ & 95 & 47,5 & 6 & 27,3 & 101 & 45,5 \\
\hline Não & 105 & 52,5 & 16 & 72,7 & 121 & 54,5 \\
\hline \multicolumn{7}{|l|}{ Número de gestações } \\
\hline Nenhuma & 21 & 10,5 & 3 & 13,6 & 24 & 10,8 \\
\hline $1-3$ & 137 & 68,5 & 17 & 77,3 & 154 & 69,4 \\
\hline 4 ou mais & 42 & 21,0 & 2 & 9,1 & 44 & 19,8 \\
\hline \multicolumn{7}{|l|}{ CD4+ (células/mm³) } \\
\hline$<200$ & 128 & 76,2 & 14 & 70,0 & 142 & 75,5 \\
\hline$\geq 200$ & 40 & 23,8 & 6 & 30,0 & 46 & 24,5 \\
\hline \multicolumn{7}{|l|}{ CD4+ Nadir (células/mm³) } \\
\hline$<200$ & 62 & 32,6 & 11 & 55,0 & 73 & 34,8 \\
\hline$\geq 200$ & 128 & 67,4 & 9 & 45,0 & 137 & 65,2 \\
\hline \multicolumn{7}{|l|}{ Uso de terapia antirretroviral altamente potente } \\
\hline Sim & 51 & 54,3 & 12 & 70,6 & 63 & 56,8 \\
\hline Não & 43 & 45,7 & 5 & 29,4 & 48 & 43,2 \\
\hline
\end{tabular}

Neg: negativo; NIC: neoplasia intraepitelial cervical. 
546,2 células $/ \mathrm{mm}^{3}$ )]. Cerca de $50 \%$ das mulheres encontrava-se em uso de terapia antirretroviral, e $56,8 \%$ delas faziam uso de HAART.

Independentemente do grau de imunossupressão, quando comparado ao padrão ouro, o exame citológico apresentou sensibilidade de 31,8\% (IC95\%: 13,9-54,9) e especificidade de 95,5\% (IC95\%: 91,6-97,9) (Tabelas 2 e 3). A captura híbrida apresentou maior sensibilidade e menor especificidade que o exame citológico [sensibilidade de 100,0\% (IC95\%: 78,0-100,0) e especificidade de 52,0\% (IC95\%: 44,8-59,1). A citologia gerou um valor preditivo positivo de 43,7\% (IC95\%: 19,7-70,1) e valor preditivo negativo de 92,7\% (IC95\%: 88,3-95,9). A captura hí- brida II, por outro lado, gerou um valor preditivo positivo de 18,6\% (IC95\%: 12,1-26,8) enquanto o valor preditivo negativo foi de $100,0 \%$ (IC95\%: 94,8-100,0). As razões de verossimilhança para o teste positivo e negativo foram estimadas em 7,1 (IC95\%: 2,9-17,1) e 0,7 (IC95\%: 0,5-0,9) para o exame citológico e em 2,1 (IC95\%: 1,8-2,4) e 0,0 para a captura híbrida II, respectivamente.

Conforme mostra a Figura 2, o uso do exame citológico implica uma maior probabilidade pós-teste quando comparado a captura híbrida II, seja qual for a probabilidade pré-teste. Sendo a probabilidade pré-teste baixa, como é o caso da prevalência da HSIL em mulheres HIV+, o exame citológico modifica de maneira mais

\begin{tabular}{|c|c|c|c|}
\hline & \multicolumn{2}{|c|}{ Teste de referência } & \multirow[t]{2}{*}{ Total } \\
\hline & $\mathrm{NIC2+}$ & $\mathrm{Neg} / \mathrm{NIC1}$ & \\
\hline \multicolumn{4}{|c|}{ Todas as mulheres $(\mathrm{N}=222)$} \\
\hline \multicolumn{4}{|l|}{ Citologia } \\
\hline HSIL+ & 7 & 9 & 16 \\
\hline HSIL- & 15 & 191 & 206 \\
\hline Total & 22 & 200 & 222 \\
\hline \multicolumn{4}{|l|}{$\mathrm{CH} \|$} \\
\hline HPVo & 22 & 96 & 118 \\
\hline $\mathrm{HPVn}$ & 0 & 104 & 104 \\
\hline Total & 22 & 200 & 222 \\
\hline \multicolumn{4}{|c|}{ Mulheres com Nadir linfócito T CD4+ $\geq 200(n=137)$} \\
\hline \multicolumn{4}{|l|}{ Citologia } \\
\hline $\mathrm{HSIL}+$ & 2 & 5 & 7 \\
\hline HSIL- & 7 & 123 & 130 \\
\hline Total & 9 & 128 & 137 \\
\hline \multicolumn{4}{|l|}{$\mathrm{CH} \|$} \\
\hline HPVo & 9 & 50 & 59 \\
\hline$H P V n$ & 0 & 78 & 78 \\
\hline Total & 9 & 128 & 137 \\
\hline \multicolumn{4}{|c|}{ Mulheres com Nadir linfócito T CD4+ < $200(n=73)$} \\
\hline \multicolumn{4}{|l|}{ Citologia } \\
\hline $\mathrm{HSIL}+$ & 4 & 3 & 7 \\
\hline HSIL- & 7 & 59 & 66 \\
\hline Total & 11 & 62 & 73 \\
\hline \multicolumn{4}{|l|}{$\mathrm{CH} \|$} \\
\hline HPVo & 11 & 40 & 51 \\
\hline HPVn & 0 & 22 & 22 \\
\hline Total & 11 & 62 & 73 \\
\hline
\end{tabular}

CH II: captura híbrida II; HPVn: vírus do papiloma humano não oncogênico; HPVo: vírus do papiloma humano oncogênico; HSIL: lesão intraepitelial escamosa de alto grau; Neg: negativo; NIC: neoplasia intraepitelial cervical.

* 12 mulheres não apresentavam resultado de linfócito T CD4+. 
Performance do exame citológico e da captura híbrida II na identificação da lesão intraepitelial escamosa de alto grau em mulheres HIV+ segundo o grau de imunossupressão indicado pelo linfócito T CD4+ Nadir.

\begin{tabular}{|c|c|c|}
\hline & Exame citológico [IC95\%] & Captura híbrida [IC95\%] \\
\hline \multicolumn{3}{|l|}{ Todas as mulheres $(\mathrm{N}=222 *)$} \\
\hline Sensibilidade & $31,8 \%[13,9-54,9]$ & $100,0 \%[78,0-100,0]$ \\
\hline Especificidade & $95,5 \%[91,6-97,9]$ & $52,0 \%[44,8-59,1]$ \\
\hline Valor preditivo positivo & $43,7 \%[19,75-70,1]$ & $18,6 \%[12,1-26,8]$ \\
\hline Valor preditivo negativo & $92,7 \%[88,3-95,9]$ & $100,0 \%[94,8-100,0]$ \\
\hline Razão de verossimilhança positiva & $7,1[2,9-17,1]$ & $2,1[1,8-2,4]$ \\
\hline Razão de verossimilhança negativa & $0,7[0,5-0,9]$ & $0,0[0,0-\mathrm{NaN}]$ \\
\hline \multicolumn{3}{|c|}{ Mulheres com contagem de Nadir linfócito T CD4+ $\geq 200$ células $/ \mathrm{mm}^{3}(n=137)$} \\
\hline Sensibilidade & $22,2 \%[2,8-60,0]$ & $100,0 \%[55,5-100,0]$ \\
\hline Especificidade & $96,1 \%[91,1-98,7]$ & $60,9 \%[51,9-69,4]$ \\
\hline Valor preditivo positivo & $28,6 \%[3,7-71,0]$ & $15,2 \%[7,2-27,0]$ \\
\hline Valor preditivo negativo & $94,6 \%[89,2-97,8]$ & $100,0 \%[93,1-100,0]$ \\
\hline Razão de verossimilhança positiva & $5,7[1,3-25,3]$ & $2,6[2,1-3,2]$ \\
\hline Razão de verossimilhança negativa & $0,8[0,6-1,1]$ & $0,0[0,0-\mathrm{NaN}]$ \\
\hline \multicolumn{3}{|c|}{ Mulheres com contagem de Nadir linfócito T CD4+ < 200 células/mm33 $(n=73$ ) } \\
\hline Sensibilidade & $36,4 \%[10,9-69,2]$ & $100,0 \%[61,5-100,0]$ \\
\hline Especificidade & $95,2 \%[86,5-99,0]$ & $35,5 \%[23,7-48,7]$ \\
\hline Valor preditivo positivo & $57,1 \%[18,4-90,1]$ & $21,6 \%[11,3-35,3]$ \\
\hline Valor preditivo negativo & $89,4 \%[79,4-95,6]$ & $100,0 \%[78,0-100,0]$ \\
\hline Razão de verossimilhança positiva & $7,5[1,9-29,1]$ & $1,5[1,3-1,9]$ \\
\hline Razão de verossimilhança negativa & $0,7[0,4-1,0]$ & $0,0[0,0-\mathrm{NaN}]$ \\
\hline
\end{tabular}

$\mathrm{NaN}$ : valor indefinido.

* 12 mulheres não apresentavam resultado de linfócito T CD4+.

significativa a probabilidade pré-teste. A Figura 3 mostra graficamente que a captura híbrida II apresenta maior sensibilidade e menor especificidade quando comparado com o exame citológico. Além disso, mostra também que a captura híbrida II prediz melhor a ausência de HSIL, enquanto o exame citológico prediz melhor a presença da HSIL.

Estratificando pelo grau de imunossupressão, nota-se que a sensibilidade, o valor preditivo positivo e a razão de verossimilhança para o teste positivo do exame citológico são maiores em mulheres que já sofreram imunossupressão grave (Tabelas 2 e 3), não sendo a diferença estatisticamente significante. No caso da captura híbrida II, a especificidade e a razão de verossimilhança para o teste positivo são estatisticamente diferentes nos estratos. A especificidade foi de $60,9 \%$ (IC95\%: 51,9-69,4) para o estrato CD4+ Nadir $\geq$ 200 células $/ \mathrm{mm}^{3}$ e de 35,5\% (IC95\%: 23,7-48,7) para o estrato CD4+ Nadir $<200$ células $/ \mathrm{mm}^{3}$. Enquanto a razão de verossimilhança para o teste positivo foi de 2,6 (IC95\%: 2,1-3,2) para o estrato
CD4+ Nadir $\geq 200$ células $/ \mathrm{mm}^{3}$ e de 1,5 (IC95\%: 1,3-1,9) para o estrato CD4+ Nadir $<200$ células / $\mathrm{mm}^{3}$ (Tabela 3).

\section{Discussão}

Neste estudo, estimamos e comparamos, para mulheres HIV+, o desempenho do exame citológico e de captura híbrida II. Observamos que a captura híbrida II se mostrou mais sensível e o exame citológico, por outro lado, se mostrou mais específico. Na literatura, as estimativas de sensibilidade e especificidade do exame citológico e da captura híbrida II em mulheres HIV+ e em mulheres em geral apresentam resultados divergentes 8,33 . Para mulheres em geral, metanálises estimam que a sensibilidade do exame citológico e da captura híbrida II aproxima-se de $55 \%$ e $90 \%$, respectivamente $34,35,36,37$. Estudos randomizados e metanálises indicam que a captura híbrida II apresenta maior sensibilidade para o rastreio de HSIL que o exame citológico 37,38. 


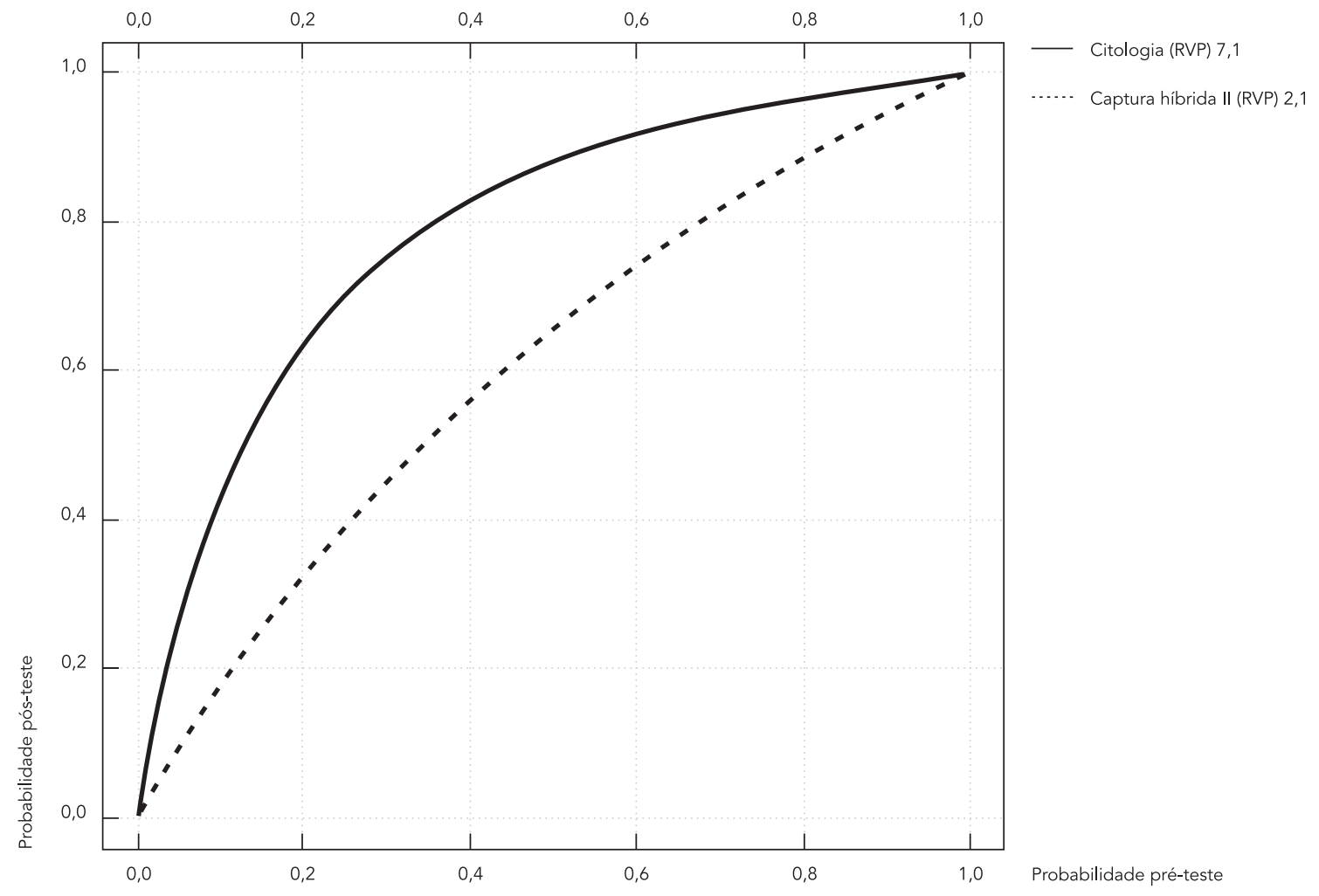

Nossos resultados confirmam esses achados para mulheres HIV+.

O exame citológico apresentou maior razão de verossimilhança para o teste positivo, o que indica que este exame tem maior capacidade preditiva para HSIL. A razão de verossimilhança para o teste positivo indica o quanto o resultado de um teste é capaz de mudar a probabilidade de se ter uma doença. Suponhamos que uma mulher HIV+ apresente exame citológico com resultado positivo para HSIL. O que pode ser inferido sobre a probabilidade de essa mulher ter HSIL? A prevalência de HSIL na coorte de mulheres HIV+ acompanhadas no IPEC/Fiocruz é de $4,1 \% 39$, o que corresponde a uma chance (ou odds) de 0,04. Multiplicando a chance de HSIL pré-teste pela razão de verossimilhança para o teste positivo, obtém-se a chance pós-teste de 0,3 . Dessa forma, a probabilidade pós-teste de HSIL é de 23,2\%. De modo análogo, o que podemos dizer sobre a chance de a mulher ter HSIL dado um resultado positivo da captura hí- brida II? Multiplicando a chance pré-teste pela razão de verossimilhança para o teste positivo, obtém-se a chance pós-teste de 0,09. Dessa forma, a probabilidade pós-teste de se ter HSIL é de $8,1 \%$. Assim, nossos resultados indicam que o resultado positivo do exame citológico muda a probabilidade pré-teste de HSIL de modo mais significativo que o resultado positivo da captura híbrida II.

Algumas medidas podem aumentar a especificidade da captura híbrida II e com isso aumentar a razão de verossimilhança para o teste positivo desse exame. A primeira medida consiste em usar tal técnica apenas em mulheres com idade superior a 30 anos. De fato, a utilização da captura híbrida II em mulheres jovens não é recomendada pela alta incidência de infecções transitórias 40 . Outra medida constitui o uso da captura híbrida II com um ponto de corte mais restritivo para o diagnóstico positivo ( $R L U>2,5$ ). Nesse estudo, utilizamos o ponto de corte de 1 , conforme recomendado pelo fabricante. 
A sensibilidade e especificidade do exame citológico e da captura híbrida II são mostradas pelo do ponto correspondente ao exame. As linhas cheias refletem as razões de verossimilhança para o teste positivo e negativo do exame citológico

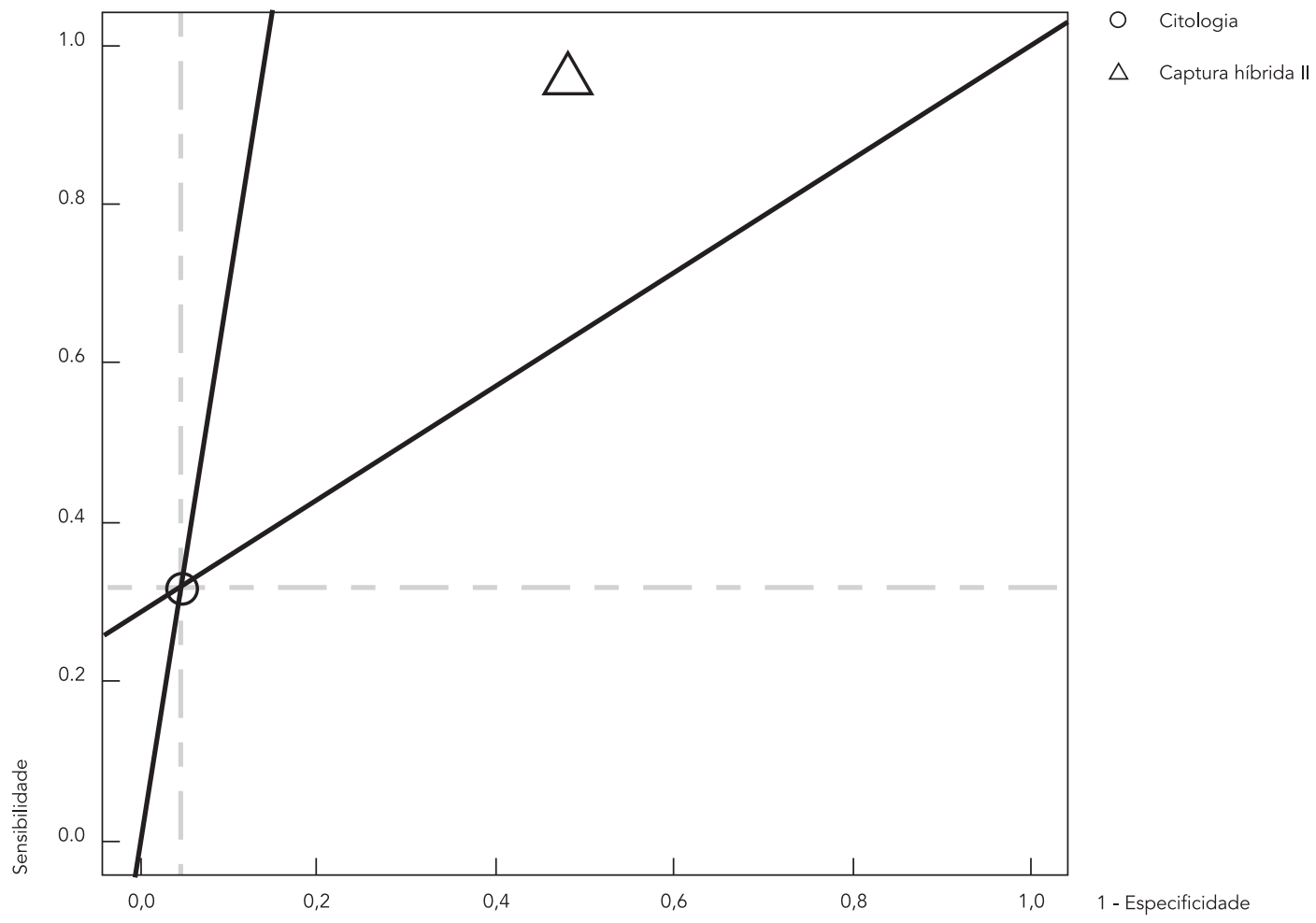

Esta análise possui limitações. Como descrito na metodologia, o médico, ao realizar o exame colposcópico, estava ciente do resultado do exame citológico. Ou seja, é possível que na presença de uma alteração citológica o médico tenha realizado o exame colposcópico com mais cuidado e que, portanto, possa ter identificado mais lesões quando comparado à realização da colposcopia na ausência de alteração citológica. Isso implica uma superestimação da sensibilidade e especificidade do exame citológico. É também possível que o mesmo viés de aferição ocorra em função do grau de imunossupressão da paciente, quando o médico seria mais cuidadoso ao avaliar uma paciente com imunossupressão avançada. Viés de seleção também pode ter influenciado os nossos achados. Conforme mostra a Figura 1, uma parcela significativa de mulheres foi excluída da análise. As mulheres que não realizaram a colposcopia dentro do prazo determinado pelo estudo foram excluídas por não mais representarem o estado da doença no momento da inclusão na coorte. As mulheres com resultado de colposcopia insatisfatório ou de lesão intraepitelial de baixo grau que não foram submetidas à avaliação histológica também foram excluídas por não ser possível saber, de fato, o diagnóstico final dessas mulheres segundo o padrão ouro usado neste estudo. Este possível viés de seleção induzido pela exclusão de uma parcela significativa das mulheres sugere cautela na interpretação do desempenho de cada teste isoladamente. Apesar desta limitação, a comparação do desempenho dos testes na mesma amostra permanece válida.

Recomendações quanto ao esquema de rastreamento das lesões precursoras do câncer do colo do útero variam entre países, assim como entre subgrupos de mulheres. A recomendação atual do Ministério da Saúde, para a população em geral, é a realização anual do exame citológico e após dois resultados negativos consecutivos, o exame passa a ser realizado a cada três anos 24. Para mulheres HIV+, a recomendação é o exame citológico semestral e após dois resulta- 
dos negativos consecutivos, o exame passa a ser realizado anualmente, não havendo diferenciação no rastreamento em função do grau de imunossupressão encontrado 41 . Nossas estimativas da capacidade diagnóstica do exame citológico e da captura híbrida II contribuem para a discussão do melhor esquema de rastreamento do câncer do colo do útero para mulheres HIV+ em nosso meio. Os exames de rastreamento exigem de modo diferenciado os profissionais de saúde com relação à capacidade técnica e tempo des- pendido ou a infraestrutura local. Logo, a análise da melhor estratégia de rastreamento para nosso contexto necessita levar em consideração múltiplos fatores, como por exemplo: a carga da doença, capacidade diagnóstica, a disponibilidade financeira para iniciar e manter uma intervenção, a infraestrutura disponível e a aceitação social e política de uma determinada intervenção. Fazse necessária uma análise de custo-efetividade do rastreamento do câncer do colo do útero em mulheres $\mathrm{HIV}+$.

\section{Resumo}

As lesões intraepiteliais escamosas de alto grau (HSIL) são precursoras do câncer do colo do útero, com maior risco de ocorrência e desenvolvimento em mulheres $H I V+$. Neste trabalho, estimamos e comparamos o desempenho do exame citológico e da captura híbrida II no rastreamento das lesões precursoras em mulheres HIV+. A população de estudo compreendeu mulheres acompanhadas na coorte prospectiva aberta do Instituto de Pesquisa Clínica Evandro Chagas da Fundação Oswaldo Cruz (IPEC/Fiocruz). A colposcopia e histologia foram consideradas conjuntamente na definição do teste de referência. O exame citológico apresentou sensibilidade de 31,8\% e especificidade de 95,5\%, enquanto a captura híbrida II apresentou maior sensibilidade (100\%) e menor especificidade (52\%). As razões de verossimilhança para o teste positivo e negativo foram estimadas em 7,1 e 0,7 para o exame citológico e em 2,1 e 0,0 para a captura híbrida II, respectivamente.

\section{Colaboradores}

L. M. Raposo, L. Velasque, P. M. Luz, T. Vanni, P. E. A. A. Brasil e C. J. Struchiner contribuíram com a análise dos dados, interpretação dos resultados, elaboração do manuscrito, e aprovaram a versão final do artigo. R. K. Friedman, F. Russomano, V. G. Veloso e B. Grinsztejn contribuíram com a aquisição dos dados, interpretação dos resultados, elaboração do manuscrito, e aprovaram a versão final do artigo. A. Cytryn e A. C. V. Andrade contribuíram com a aquisição dos dados e aprovaram a versão final do artigo.

\section{Agradecimentos}

Conselho Nacional de Desenvolvimento Científico e Tecnológico (CNPq) e Fundação de Amparo à Pesquisa do Estado do Rio de Janeiro (FAPERJ). 


\section{Referências}

1. Snijders P, Steenbergen R, Heideman D, Meijer C. HPV-mediated cervical carcinogenesis: concepts and clinical implications. J Pathol 2006; 208: 152-64.

2. Bosch FX, de Sanjose S. The epidemiology of human papillomavirus infection and cervical cancer. Dis Markers 2007; 23:213-27.

3. Schiffman M, Castle P, Jeronimo J, Rodriguez A Wacholder S. Human papillomavirus and cervical cancer. Lancet 2007; 370:890-907.

4. Wright TJ. Cervical cancer screening in the $21 \mathrm{st}$ century: is it time to retire the PAP smear? Clin Obstet Gynecol 2007; 50:313-23.

5. Sawaya G, Brown A, Washington A, Garber A. Clinical practice. Current approaches to cervical-cancer screening. N Engl J Med 2001; 344:1603-7.

6. Franceschi S, Jaffe H. Cervical cancer screening of women living with HIV infection: a must in the era of antiretroviral therapy. Clin Infect Dis 2007; 45:510-3.

7. Palefsky J. Human papillomavirus-related tumors in HIV. Curr Opin Oncol 2006; 18:463-8.

8. Heard I. Prevention of cervical cancer in women with HIV. Curr Opin HIV AIDS 2009; 4:68-73.

9. Palefsky JM. Cervical human papillomavirus infection and cervical intraepithelial neoplasia in women positive for human immunodeficiency virus in the era of highly active antiretroviral therapy. Curr Opin Oncol 2003; 15:382-8.

10. Strickler HD, Burk RD, Fazzari M, Anastos K, Minkoff H, Massad LS, et al. Natural history and possible reactivation of human papillomavirus in human immunodeficiency virus-positive women. J Natl Cancer Inst 2005; 97:577-86.

11. Clifford G, Polesel J, Rickenbach M, Dal Maso L, Keiser O, Kofler A, et al. Cancer risk in the Swiss HIV Cohort Study: associations with immunodeficiency, smoking, and highly active antiretroviral therapy. J Natl Cancer Inst 2005; 97:425-32.

12. Bonnet F, Chêne G. Evolving epidemiology of malignancies in HIV. Curr Opin Oncol 2008; 20: 534-40.

13. Quinn T. HIV epidemiology and the effects of antiviral therapy on long-term consequences. AIDS 2008; 22 Suppl 3:S7-12.

14. Campos D, Ribeiro S, Grinsztejn B, Veloso V, Valente J, Bastos F, et al. Survival of AIDS patients using two case definitions, Rio de Janeiro, Brazil, 1986-2003. AIDS 2005; 19 Suppl 4:S22-6.

15. Fonseca M, Bastos F. Twenty-five years of the AIDS epidemic in Brazil: principal epidemiological findings, 1980-2005. Cad Saúde Pública 2007; 23 Suppl 3:S333-44.

16. Patel P, Hanson D, Sullivan P, Novak R, Moorman A, Tong $\mathrm{T}$, et al. Incidence of types of cancer among HIV-infected persons compared with the general population in the United States, 1992-2003. Ann Intern Med 2008; 148:728-36.

17. Heard I, Tassie JM, Kazatchkine MD, Orth G. Highly active antiretroviral therapy enhances regression of cervical intraepithelial neoplasia in HIV-seropositive women. AIDS 2002; 16:1799-802.
18. Xi LF, Kiviat NB. Cervical neoplasia and highly active antiretroviral therapy. J Natl Cancer Inst 2004; 96:1051-3.

19. Mangclaviraj S, Kerr SJ, Chaithongwongwatthana S, Ananworanich J, Hirschel B, Emery S, et al. Nadir CD4 count and monthly income predict cervical squamous cell abnormalities in HIV-positive women in a resource-limited setting. Int J STD AIDS 2008; 19:529-32.

20. Stival C, Lazzarotto M, Rodrigues Y, Vargas V. Avaliação comparativa da citopatologia positiva, colposcopia e histopatologia: destacando a citopatologia como método de rastreamento da neoplasia do colo do útero. Rev Bras Anal Clin 2005; 37:215-8.

21. Santos A, Derchain S, Calvert E, Martins M, Dufloth M, Martinez E. Desempenho do exame colpocitológico com revisão por diferentes observadores e da captura híbrida II no diagnóstico da neoplasia intraepitelial cervical graus 2 e 3. Cad Saúde Pública 2003 ; 19:1029-37.

22. Longatto-Filho A, Erzen M, Branca M, Roteli-Martins C, Naud P, Derchain S, et al. Human papillomavirus testing as an optional screening tool in low-resource settings of Latin America: experience from the Latin American Screening study. Int J Gynecol Cancer 2006; 16:955-62.

23. Tuon FFB, Bittencourt M, Panichi M, Pinto A. Avaliação da sensibilidade e especificidade dos exames citopatológico e colposcópico em relação ao exame histológico na identificação de lesões intraepiteliais cervicais. Rev Assoc Méd Bras 2002; 48:140-4.

24. Instituto Nacional de Câncer. Nomenclatura brasileira para laudos cervicais e condutas preconizadas: recomendações para profissionais de saúde. J Bras Patol Med Lab 2006; 42:351-73.

25. Grinsztejn B, Bastos F, Veloso V, Friedman R, Pilotto J, Schechter M, et al. Assessing sexually transmitted infections in a cohort of women living with HIV/AIDS, in Rio de Janeiro, Brazil. Int J STD AIDS 2006; 17:473-8.

26. Grinsztejn B, Veloso V, Levi J, Velasque L, Luz P, Friedman R, et al. Factors associated with increased prevalence of human papillomavirus infection in a cohort of HIV-infected Brazilian women. Int J Infect Dis 2009; 13: 72-80.

27. The 1988 Bethesda System for reporting cervical/ vaginal cytological diagnoses. National Cancer Institute Workshop. JAMA 1989; 262:931-4.

28. Walker P, Dexeus S, De Palo G, Barrasso R, Campion M, Girardi F, et al. International terminology of colposcopy: an updated report from the International Federation for Cervical Pathology and Colposcopy. Obstet Gynecol 2003; 101:175-7.

29. Medronho RA, Perez MA. Testes diagnósticos. In: Medronho RA, Carvalho AM, Bloch KV, Luiz RR, Werneck GL, organizadores. Epidemiologia. São Paulo: Atheneu; 2002. p. 259-70.

30. Biggerstaff BJ. Comparing diagnostic tests: a simple graphic using likelihood ratios. Stat Med 2000; 19:649-63. 
31. Stevenson M. epi R: functions for analysing epidemiological data. http://cran.r-project.org/web/ packages/epiR/index.html.

32. Brasil P. DiagnosisMed: diagnostic test accuracy evaluation for medical professionals. http://cran.rproject.org/web/packages/DiagnosisMed/.

33. Anderson J, Paramsothy P, Heilig C, Jamieson D, Shah K, Duerr A. Accuracy of Papanicolaou test among HIV-infected women. Clin Infect Dis 2006; 42:562-8.

34. Arbyn M, Bergeron C, Klinkhamer P, Martin-Hirsch P, Siebers A, Bulten J. Liquid compared with conventional cervical cytology: a systematic review and meta-analysis. Obstet Gynecol 2008; 111: 167-77.

35. Cong X, Cox D, Cantor S. Bayesian meta-analysis of Papanicolaou smear accuracy. Gynecol Oncol 2007; 107:S133-7.

36. Cuzick J, Clavel C, Petry K, Meijer C, Hoyer H, Ratnam S, et al. Overview of the European and North American studies on HPV testing in primary cervical cancer screening. Int J Cancer 2006; 119: 1095-101.

37. Koliopoulos G, Arbyn M, Martin-Hirsch P, Kyrgiou M, Prendiville W, Paraskevaidis E. Diagnostic accuracy of human papillomavirus testing in primary cervical screening: a systematic review and metaanalysis of non-randomized studies. Gynecol Oncol 2007; 104:232-46.
38. Arbyn M, Buntinx F, Van Ranst M, Paraskevaidis E, Martin-Hirsch P, Dillner J. Virologic versus cytologic triage of women with equivocal Pap smears: a meta-analysis of the accuracy to detect high-grade intraepithelial neoplasia. J Natl Cancer Inst 2004; 96:280-93.

39. Luz P, Velasque L, Friedman R, Russomano F, Andrade A, Moreira R, et al. Cervical cytological abnormalities and factors associated with high-grade squamous intra-epithelial lesions among HIV-infected women from Rio de Janeiro, Brazil. Int J STD AIDS; no prelo.

40. Dillner J, Rebolj M, Birembaut P, Petry K, Szarewski A, Munk C, et al. Long term predictive values of cytology and human papillomavirus testing in cervical cancer screening: joint European cohort study. BMJ 2008; 337:a1754

41. Programa Nacional de DST e AIDS. Recomendações para terapia antirretroviral em adultos infectados pelo HIV: 2008. Brasília: Ministério da Saúde; 2008.

Recebido em 20/Abr/2010

Versão final reapresentada em 08/Mar/2011

Aprovado em 26/Abr/2011 